\title{
A COMPUTER-ASSISTED PROOF OF THE FEIGENBAUM CONJECTURES
}

\author{
BY OSCAR E. LANFORD III ${ }^{1}$
}

1. Introduction. Let $M$ denote the space of continuously differentiable even mappings $\psi$ of the interval $[-1,1]$ into itself such that

M1. $\psi(0)=1$,

M2. $x \psi^{\prime}(x)<0$ for $x \neq 0$.

M2 says that $\psi$ is strictly increasing on $[-1,0)$ and strictly decreasing on $(0,1]$, so $M$ is a space of mappings which are unimodal in a strict sense.

Condition M1 says that the unique critical point 0 is mapped to 1 . We want to consider $\psi$ 's which map 1 slightly - but not too far - to the left of 0 . It may then be possible to find nonoverlapping intervals $I_{0}$ about 0 and $I_{1}$ near 1 which are exchanged by $\psi$. Technically, we proceed as follows: Write $a$ for $-\psi(1)=$ $-\psi^{2}(0)$ and $b$ for $\psi(a)$; we suppress from the notation the dependence of $a$ and $b$ on $\psi$. Define $D(T)$ to be the set of all $\psi$ 's in $M$ such that:
D1. $a>0$,
D2. $b>a$,
D3. $\psi(b) \leqslant a$.

The two intervals $I_{0}=[-a, a]$ and $I_{1}=[b, 1]$ are then nonoverlapping and $\psi$ maps $I_{0}$ into $I_{1}$ and vice versa. If $\psi \in O(T)$, then $\left.\psi \circ \psi\right|_{I_{0}}$ has a single critical point, which is a minimum. By making the change of variables $x \rightarrow-a x$, we replace $I_{0}$ by $[-1,1]$ and the minimum by a maximum, i.e., if we define

$$
T \psi(x)=-\frac{1}{a} \psi \circ \psi(-a x) \text { for } x \in[-1,1]
$$

then $T \psi$ is again in $M$. Thus, $T$ defines a mapping of $D(T)$ into $M$. (In general, $T \psi$ need not lie in $O(T)$. If $a$ is small, then $T \psi(1)$ will be approximately 1 so $T \psi$ will not satisfy D1. On the other hand, if $\psi(b)$ is near $a$, then $T \psi(1)$ will be near -1 from which it follows that $T \psi$ does not satisfy D2.)

M. Feigenbaum [6] has proposed an explanation for some universal features displayed by infinite sequences of period doubling bifurcations based on some conjectures about $T$. We will not review has argument here; a version with due regard for mathematical technicalities may be found in Collet and Eckmann [3],

Received by the editors October 27, 1981.

1980 Mathematics Subject Classification. Primary 58F14.

1 The author gratefully acknowledges the financial support of the Stiftung Volkswagenwerk for a visit to the IHES during which this paper was written, and the continuing financial support of the National Science Foundation (Grant MCS 78-06718). 
Collet, Eckmann and Lanford [4], or in Lanford [8]. The purpose of this note is to announce a proof of essentially all of these conjectures and to indicate the kind of analysis used.

\section{Statement of results.}

THEOREM 1. There exists a function $g$, analytic and even on $\{z \in \mathrm{C}:|z|$ $<\sqrt{8}\}$ whose restriction to $[-1,1]$ is a fixed point for $T$. The Schwarzian derivative of $g$ is negative on $[-1,1]$.

Let $\Omega$ denote $\left\{z \in \mathbf{C}:\left|z^{2}-1\right|<2.5\right\}$ and write

$\mathfrak{S}$ for the Banach space of even functions bounded and analytic on $\Omega$, real on real points, equipped with the supremum norm.

$\mathfrak{S}_{0}$ for the subspace of $\mathfrak{S}$ consisting of those functions vanishing (to second order) at 0 .

$\mathfrak{S}_{1}$ for $\mathfrak{S}_{0}+1$.

Proposition 2. There is an open neighborhood $V$ of $g$ in $\mathfrak{S}_{1}$ such that Every $\psi \in V$ is in $D(T)$ (i.e., its restriction to $[-1,1]$ is).

If $\psi \in V, T \psi \in \mathfrak{S}_{1}$.

$T$ is infinitely differentiable as a mapping from $V$ into $\mathfrak{S}_{1}$,

The derivative DT( $\psi)$ is compact operator on $\mathfrak{S}_{0}$ for each $\psi \in V$.

THEOREM 3. DT $(g)$ is hyperbolic on $\mathfrak{S}_{0}$ with one-dimensional expanding subspace; the expanding eigenvalue $\delta$ is positive.

In other words: The spectrum of $D T(g)$ does not intersect the unit circle, and the part of the spectrum outside the unit circle consists of a single simple positive eigenvalue $\delta$.

It then follows from invariant manifold theory that $T$ admits locally invariant local stable and local unstable manifolds, of codimension one and dimension one respectively. Because of the noninvertibility of $T$, we do not construct global stable and unstable manifolds; we will let $W_{s}$ and $W_{u}$ denote respectively some particular local stable and local unstable manifolds.

Let $\Sigma_{0}$ denote the bifurcation surface for the simple period-doubling bifurcation. By this we mean the following: Any $\psi$ in $M$ has exactly one fixed point $x_{0}$ in $[0,1] ; \Sigma_{0}$ then denotes $\left\{\psi \in M: \psi^{\prime}\left(x_{0}\right)=-1 ;(\psi \circ \psi)^{\prime \prime \prime}\left(x_{0}\right)<0\right\}$. As a one-parameter family of $\psi$ 's crosses $\Sigma_{0}$ (in the appropriate direction) the fixed point $x_{0}$ loses stability in favor of an attracting orbit of period 2.

THEOREM 4. There is a positive integer $j$ and an element $g_{j}^{*}$ of $W_{u}$ such that $T^{j} g_{j}^{*} \in \Sigma_{0}$.

Except for the difficulties in defining a global unstable manifold, we could formulate this theorem by saying that the unstable manifold crosses $\Sigma_{0}$. We 
would like to know more, viz., that the crossing is transversal. This - properly formulated - is almost certainly true, but we have not proved it.

Let $\psi_{\mu}^{(0)}(x)$ denote the quadratic mapping $1-\mu x^{2}$.

THEOREM 5. There is a positive integer $j$ and a parameter value $\mu_{\infty}$ (between 1.4011550 and 1.4011554) such that $\psi_{\mu}^{(0)}$ is in $D\left(T^{j}\right)$ for $\mu$ sufficiently near to $\mu_{\infty}$ and such that the curve $T^{j} \psi_{\mu}^{(0)}$ crosses $W_{s}$ transversally at $\mu=\mu_{\infty}$.

Except for technicalities, this says that $\psi_{\mu}^{(0)}$ crosses the stable manifold transversally at $\mu_{\infty}$.

3. Remarks on the method of proof. The heart of the proof is a set of complicated numerical estimates proved rigorously with the aid of a computer. To formulate these estimates, we have first to establish some notation. We will work, initially, not in $\mathfrak{S}_{1}$ but in a subspace equipped with a stronger norm. The idea is that we want to write $\psi$ as

$$
\psi(x)=1-x^{2} h\left(x^{2}\right)
$$

and to use the $l^{1}$ norm for the Taylor coefficients of $h$ at 1 . Formally, given an element $(u, v)$ of $\mathbf{R} \oplus l^{1}$, we associate with it an element $\psi$ of $\mathfrak{S}_{\mathbf{1}}$ by

$$
\psi(x)=1-x^{2}\left\{u / 10+\sum_{n=1}^{\infty} v_{n}\left(\frac{\left(x^{2}-1\right)}{2.5}\right)^{n}\right\}
$$

We denote the set of $\psi$ 's obtained in this way by $A$, and we equip $A$ with the norm $|u|+\Sigma\left|v_{n}\right|$. Note that $A$ contains any element of $\mathfrak{S}_{1}$ which is analytic on the closure of $\Omega$. (Of course, $R \oplus l^{1}$ could have been identified with $l^{1}$, but we have singled out the $u$ component - and introduced the factor of 10 in the formula (3.1) for $\psi(x)$ - for convenience later on.) For the remainder of this section, the norm of an element of $A$ will always mean the norm of $l^{1}$ type just introduced.

The first step is to choose an explicit polynomial $\psi_{0}$ which will turn out to be a good approximate fixed point. We will take $\psi_{0}$ to be the polynomial of degree 20 defined by the first ten terms of the series given in Table 1 below. It can be checked without difficulty that

For any $\psi \in A$ with $\left\|\psi-\psi_{0}\right\|<.01, T \psi \in A$

$T$ is infinitely differentiable from $\left\{\left\|\psi-\psi_{0}\right\|<.01\right\}$ to $A$.

For any $\psi$ in this ball, $D T(\psi)$ is a compact operator on $A$.

Identifying $A$ with $\mathbf{R} \oplus l^{1}$, we can represent $D T(\psi)$ as a matrix

$$
\left(\begin{array}{ll}
\alpha(\psi) & \beta(\psi) \\
\gamma(\psi) & \delta(\psi)
\end{array}\right)
$$


with $\alpha \in \mathbf{R} ; \beta \in\left(l^{1}\right)^{*} ; \gamma \in l^{1} ; \delta \in L\left(l^{1}, l^{1}\right)$. In this notation, we can formulate

Estimate 1 . If $\left\|\psi-\psi_{0}\right\|<.01$, then

$$
|\alpha-4.669|<.148 ; \quad\|\beta\|<.560 ; \quad\|\gamma\|<.756 ; \quad\|\delta\|<.719 .
$$

These bounds imply that the inequality

$$
[\alpha(\psi)-1][1-\|\delta(\psi)\|]>\|\beta(\psi)\| \cdot\|\gamma(\psi)\|
$$

holds uniformly on the ball of radius .01 about $\psi_{0}$. If $T$ has a fixed point $g$ in this ball, then hyperbolicity of $D T(g)$ acting on A follows readily from (3.2).

To prove the existence of a fixed point, we use a variant of Newton's method. Instead of studying

$$
\psi \mapsto \psi-(D T(\psi)-\mathbb{1})^{-1}[T \psi-\psi],
$$

we replace $(D T(\psi)-\mathbb{1})^{-1}$ by the approximation

$$
J=\left(\begin{array}{cc}
\frac{1}{3.669} & 0 \\
0 & -\mathbb{1}
\end{array}\right),
$$

and we apply the contraction mapping principle to the mapping

$$
\psi \mapsto \Phi(\psi)=\psi-J \cdot[T \psi-\psi]
$$

which has the same fixed points as $T$.

A simple calculation using Estimate 1 shows that

$$
\|D \Phi(\psi)\|<.9 \text { for }\left\|\psi-\psi_{0}\right\|<.01 .
$$

It will then follow from the contraction mapping theorem that $\Phi$ has a fixed point in this ball provided that

$$
\frac{\left\|\Phi\left(\psi_{0}\right)-\psi_{0}\right\|}{1-.9}<.01
$$

For this we have

ESTIMATE 2.

$$
\left\|\Phi\left(\psi_{0}\right)-\psi_{0}\right\|<4 \times 10^{-6} .
$$

Thus $T$ has a fixed point in $A$, and $D T$ at the fixed point, acting on $A$, has the hyperbolicity properties stated in Theorem 3 . Domains of analyticity may be enlarged using the functional equation for $g$, and in this way we arrive at Theorems 1 and 3 as formulated.

Furthermore, Estimate 1 makes it possible to establish the existence of a system of expanding and contracting cones for $T$ on $\left\{\psi:\left\|\psi-\psi_{0}\right\|<.01\right\}$, which in turn makes it possible to construct local stable and unstable manifolds which are not too small. This facilitates the proofs of Theorems 4 and 5 . 
The proofs of Estimates 1 and 2 are completely straightforward, if long. Consider, for example, Estimate 1. Since A is essentially $l^{1}$, we can think of $D T(\psi)$ as an infinite matrix. Norms of matrices acting on $l^{1}$ are easy to compute in terms of the matrix elements. Any matrix element can be expressed in terms of $\psi$. All but finitely many of these matrix elements are estimated analytically. For the remainder, strict upper and lower bounds are computed numerically from bounds on the Taylor coefficients for $\psi$. The arithmetic operations are performed in finite precision floating point arithmetic; the methods of interval arithmetic are used to control the effect of round-off error.

4. Supplementary remarks. 1. The results described here are descendants of (and improvements on) the results announced in [7]. Since that announcement, a completely different proof for the existence of $g$ has been given by Campanino, Epstein, and Ruelle [1].

2, The approach to proving Theorem 1 outlined in the preceding section produces strict bounds on the difference between an approximate fixed point and the exact one. These estimates can be applied to higher precision calculations. Let

$$
g^{(0)}(x)=1+\sum_{n=1}^{40} g_{n}^{(0)} x^{2 n}
$$

where the $g_{n}^{(0)}$ are given by Table 1 .

We then have strict bounds

$$
\left|g(z)-g^{(0)}(z)\right| \leqslant \begin{array}{ll}
1.5 \times 10^{-23} & \text { for }|z|^{2} \leqslant 1.5 \\
5.5 \times 10^{-13} & \text { for }|z|^{2} \leqslant 2 \\
5 \times 10^{-7} & \text { for }|z|^{2} \leqslant 6 \\
1.7 \times 10^{-2} & \text { for }|z|^{2} \leqslant 8
\end{array}
$$

These bounds are probably very conservative.

3. The domain $\Omega$ used in the statements of Proposition 2 and Theorem 3 was chosen for convenience. Many other domains, including arbitrarily small open neighborhoods of $[-1,1]$, could have been used instead. The hyperbolicity statement of Theorem 3 is formally stronger for small domains than for larger ones. (For $\Omega_{1} \subset \Omega_{2}$, any eigenfunction for $D T(g)$ on $\Omega_{2}$ is also an eigenfunction on $\left.\Omega_{1}\right)$. It can be shown, however, that any function analytic on a neighborhood of $[-1,1]$ and satisfying there the formal functional equation for an eigenvector of $D T(g)$ is actually analytic and bounded on the domain $\Omega$.

4. It follows easily from the functional equation for $g$ that $g$ is analytic on a neighborhood of the whole real axis. H. Epstein (private communication) has observed that a similar argument shows that it is analytic on a neighborhood 


\begin{tabular}{|c|c|}
\hline $\mathrm{n}$ & $g_{n}^{(0)}$ \\
\hline 1 & -1.527632997036301454035890310240 \\
\hline 2 & 0.104815194787303733216742613801 \\
\hline 3 & $0.026705670525193 \quad 354032652094944$ \\
\hline 4 & -0.003527409660908709170234190769 \\
\hline 5 & 0.000081600966547531745172190486 \\
\hline 6 & 0.000025285084233963536176262552 \\
\hline 7 & $-2.5563171662784938463532541 \times 10^{-6}$ \\
\hline 8 & $-9.6512715508912032163725768 \times 10^{-8}$ \\
\hline 9 & $2.8193463974504091370756629 \times 10^{-8}$ \\
\hline 10 & $-2.77305116079901172437 \times 10^{-10}$ \\
\hline 11 & $-3.02842702213056632983 \times 10^{-10}$ \\
\hline 12 & $2.67058928074807555396 \times 10^{-11}$ \\
\hline 13 & $9.96229164102848231059 \times 10^{-13}$ \\
\hline 14 & $-3.62420298290415608455 \times 10^{-13}$ \\
\hline 15 & $2.17965774482707047701 \times 10^{-14}$ \\
\hline 16 & $1.52923289948096260560 \times 10^{-15}$ \\
\hline 17 & $-3.184728789952775 \times 10^{-16}$ \\
\hline 18 & $1.134672106211871 \times 10^{-17}$ \\
\hline 19 & $1.881676056825439 \times 10^{-18}$ \\
\hline 20 & $-2.275612564632121 \times 10^{-19}$ \\
\hline 21 & $-9.822447629421762 \times 10^{-22}$ \\
\hline 22 & $2.064129756004508 \times 10^{-21}$ \\
\hline 23 & $-1.249320059243689 \times 10^{-22}$ \\
\hline 24 & $-1.0770612046 \times 10^{-23}$ \\
\hline 25 & $1.8727468082 \times 10^{-24}$ \\
\hline 26 & $-2.5777082101 \times 10^{-26}$ \\
\hline 27 & $-1.5541904560 \times 10^{-26}$ \\
\hline 28 & $1.2804434650 \times 10^{-27}$ \\
\hline 29 & $5.5850587986 \times 10^{-29}$ \\
\hline 30 & $-1.5278346925 \times 10^{-29}$ \\
\hline 31 & $5.0417426639 \times 10^{-31}$ \\
\hline 32 & $1.0165368070 \times 10^{-31}$ \\
\hline 33 & $-1.00690 \times 10^{-32}$ \\
\hline 34 & $-5.24253 \times 10^{-34}$ \\
\hline 35 & $1.72437 \times 10^{-34}$ \\
\hline 36 & $-1.31439 \times 10^{-35}$ \\
\hline 37 & $-1.85830 \times 10^{-38}$ \\
\hline 38 & $8.05506 \times 10^{-38}$ \\
\hline 39 & $-6.26717 \times 10^{-39}$ \\
\hline 40 & $1.76882 \times 10^{-40}$ \\
\hline
\end{tabular}

TABLE 1. 
of the imaginary axis. On the other hand, it is essentially certain that $g$ is not entire. Indeed, it appears - but has not been proved - that the singularities of $g$ nearest to the origin occur at a set of 4 periodic points of period 2 for $z \mapsto$ $g(-\lambda z), \lambda=-g(1)$, located approximately at

$$
z^{2}=-3.8428 \pm \text { i } 9.8215 \text {. }
$$

5. Proposition 2 and Theorem 3 remain true if the requirement that $\psi$ be even is dropped. In other words: No new expanding eigenvectors are introduced if we let $D T(g)$ act on functions which are not necessarily even (but which vanish to second order at 0 ).

6. Theorem 4 can be extended considerably. To formulate the extension, we need the theory of kneading sequences for unimodal mappings as developed, for example, in Chapter III.1 of Collet-Eckmann [3]. Let $\underline{K}$ be a finite kneading sequence. Except for the simple case $\underline{K}=R C$, there are associated with $\underline{K}$ three hypersurfaces in $M$ :

The set of superstable $\psi$ 's with kneading sequence $\underline{K}$.

The saddle-node or period-doubling bifurcation surface where the attracting periodic orbit passing through the critical point on the preceding surface appears.

The period-doubling bifurcation surface where that periodic orbit becomes unstable.

It can be shown that, intuitively, the unstable manifold crosses these three surfaces for each $K$; a precise version of this statement must be formulated with the same circumspection as Theorem 4. There is no reason to doubt that these crossings are all transverse.

A simple argument using the apparatus developed in [3] reduces the proof of Theorem 4 and the above extension to establishing the existence, on the local unstable manifold, of one point whose kneading sequence strictly precedes, and one whose kneading sequence strictly follows, that of $g$ (in the combinatorial ordering for kneading sequences). The proof proceeds by finding with sufficient precision two points on the unstable manifold and computing initial segments of their kneading sequences.

7. Although done by computer, the computations involved in proving the results stated are just on the boundary of what it is feasible to verify by hand. I estimate that a carefully chosen minimal set of estimates sufficient to prove Theorems 1 and 3 could be carried out, with the aid only of a nonprogrammable calculator, in a few days.

ACKNOWLEDGEMENTS. It is a pleasure for me to thank:

P. Collet, J. P. Eckmann, H. Epstein, D. Ruelle, and S. Smale for helpful discussions and encouragement.

L. Michel for his assistance in making available the computing facilities needed to carry out this work. 
Director N. Kuiper for his very gracious hospitality at the IHES.

The Stiftung Volkswagenwerk for financial support during my visit to the IHES.

The National Science Foundation for continuing financial support under Grant MCS 78-06718.

\section{REFERENCES}

1. M. Campanino, H. Epstein and D. Ruelle, On Feigenbaum's functional equation, (IHES preprint $\mathrm{P} / 80 / 32$ (1980)) Topology (to appear).

2. M. Campanino and H. Epstein, On the existence of Feigenbaum's fixed point, (IHES preprint P/80/35 (1980)) Comm. Math. Phys. (1981), 261-302.

3. P. Collet and J. P. Eckmann, Iterated maps of the interval as dynamical systems, Birkhäuser, Boston-Basel-Stuttgart, 1980.

4. P. Collet, J. P. Eckmann and O. E. Lanford, Universal properties of maps on an interval, Comm. Math. Phys. 76 (1980), 211-254.

5. M. Feigenbaum, Quantitative universality for a class of non-linear transformations, J. Statist. Phys. 19 (1978), 25-52.

6. The universal metric properties of non-linear transformations, J. Statist. Phys. 21 (1979), 669-706.

7. O. E. Lanford, Remarks on the accumulation of period-doubling bifurcations, Mathematical Problems in Theoretical Physics, Lecture Notes in Physics, vol. 116, SpringerVerlag, Berlin and New York, 1980, pp. 340-342.

8. - Smooth transformations of intervals, Séminaire Bourbaki, 1980/81, No. 563, Lecture Notes in Math., vol. 901, Springer-Verlag, Berlin, Heidelberg and New York, 1981, pp. 36-54.

INSTITUT DES HAUTES ETUDES SCIENTIFIQUES, 35, ROUTE DE CHARTRES, 91440, BURES-SUR-YVETTE, FRANCE

DEPARTMENT OF MATHEMATICS, UNIVERSITY OF CALIFORNIA, BERKELEY, CALIFORNIA 94720 (Current address) 